\title{
Effect of HMB on Phase Angle of Maintenance Hemodialysis Patients
}

\author{
Liang Qingyue, Wang Zhong*, Zong Xueping, Diao Yanqing, Cai Ping, Li Honglie, Hu Liping \\ Department of Clinical Nutrition, Chengdu Fifth People's Hospital, Chengdu, China \\ Email address: \\ 527540321@qq.com (Liang Qingyue),347005@qq.com (Wang Zhong), 397644886@qq.com (Zong Xueping), \\ 869992@qq.com (Diao Yanqing), 1951043528@qq.com (Cai Ping), 9522226744@qq.com (Li Honglie),690011070@qq.com (Hu Liping) \\ ${ }^{*}$ Corresponding author
}

\section{To cite this article:}

Liang Qingyue, Wang Zhong, Zong Xueping, Diao Yanqing, Cai Ping, Li Honglie, Hu Liping. Effect of HMB on Phase Angle of Maintenance Hemodialysis Patients. Science Discovery. Vol. 9, No. 4, 2021, pp. 150-154. doi: 10.11648/j.sd.20210904.14

Received: March 6, 2021; Accepted: June 2, 2021; Published: June 4, 2021

\begin{abstract}
Objective To observe and evaluate the effect of $\beta$ - hydroxy- $\beta$ - methylbutyric acid (HMB) on phase angle (PA) in maintenance hemodialysis (MHD) patients. Methods From March 2018 to August 2019, 64 patients who received maintenance hemodialysis, at Chengdu Fifth People's Hospital, were selected as subjects, and they were divided into experimental group and control group with single center, random, single dose, single blind control design, and 32 patients in each group. HMB intervention was performed in the trial group and placebo in the control group for 12 weeks. Human component analysis (Inbody S770) was performed before and after intervention in the two groups to observe the change of phase angle. Scored Patient-Generated Subjective Global Assessment (SGA) and Malnutrition Inflammatory Score (MIS) were used to assess the nutritional status of MHD patients before and after group entry, to observe the correlation of its numerical change and the phase angle. Results The PA, SGA, MIS of the experimental group was significantly higher $(p<0.05)$ than that of the control group $(p<0.05)$, and the test group increased better than the control group $(p<0.05)$. Positive correlation between PA and SGA, MIS $(\mathrm{p}<0.05)$. Conclusions By giving patients complementary HMB, to improve patient nutritional status, to improve the patient phase angle, and improve the patient prognosis.
\end{abstract}

Keywords: Phase Angle, $\beta$ - hydroxy- $\beta$-methyl Butyric Acid, Maintenance Hemodialysis

\section{HMB对维持性血液透析患者相位角的影响}

梁清月, 王仲", 宗雪萍, 入燕青, 蔡萍, 李轰烈, 胡利苹

成都市第五人民医院临床营养科, 成都, 中国

邮箱

527540321@qq.com (梁清月)，347005@qq.com（王仲），397644886@qq.com（宗雪萍），869992@qq.com（フ燕青）, 1951043528@qq.com(蔡萍),9522226744@qq.com(李轰烈),690011070@qq.com（胡利苹）

摘要：目的：观察和评价 $\beta$-差基- $\beta$-甲基丁酸（HMB）对维持性血液透析（MHD）患者相位角（PA）的影响。方法: 选取2018年3月-2019年8月在成都市第五人民医院接受维持性血液透析的64例患者作为研究对象, 采用单中心、随机、 单剂量、单盲对照设计, 将其分为试验组与对照组, 每组32例。试验组予以HMB进行干预, 对照组予以安慰剂, 共12 周。两组患者干预前后进行人体成分分析（Inbody S770），观察其相位角的变化。入组前后分别使用主观全面评定法 (SGA) 和营养不良炎症评分（MIS）评估MHD患者的营养状况，观察其数值变化与相位角的相关性。结果：与治疗 前比较, 试验组干预后PA、SGA、MIS均较干预前明显升高（ $\mathrm{P}<0.05 ）$ ，且试验组升高幅度优于对照组（ $\mathrm{P}<0.05 ）$; $\mathrm{PA}$ 与 SGA、MIS均正相关性 $(\mathrm{P}<0.05)$ 。结论：通过对维持性血液透析患者给予补充 $\mathrm{HMB}$, 改善患者营养状况, 提高 患者相位角, 改善患者预后。 
关键词: 相位角, $\beta$-羟基- $\beta$-甲基丁酸, 维持性血液透析

\section{1. 引言}

由于疾病伴随的厌食、胃肠道功能障碍、代谢性酸 中毒和持续的微炎性状态等因素 [1], 维持性血液透析患 者营养不良患病率高达 $20 \% \sim 70 \%[2]$ 。众所周知, 营养不 良增加患者的住院时间、并发症发生率、病死率、再住 院费用和医疗费用 [3]。因此, 如何有效的预测和识别 MHD患者的营养不良, 早期进行营养评估, 并开展适当 的营养干预, 已经成为慢性肾病综合管理的重要组成部 分 [4]。HMB 是 $\beta$-羟基- $\beta$-甲基丁酸 ( $\beta$ - hydroxy- $\beta$-methyl butyric acid) 是必需氨基酸亮氨酸在体内通过其代谢产 物（ $\alpha$ 酮异己酸, $\alpha$-KIC) 产生的衍生物 [5], HMB 是作为 安全、有效的营养补充剂[6], 可以改善MHD患者营养状 况。

相位角 (PA) 是由生物电阻抗技术衍生而出的一项评 估营养状况和预后的良好客观指标[7]。PA值可早期发现维 持性血透患者的营养不良 [8], 准确评估血透患者残余肾功 能[9]。此研究期望可通过添加HMB改善MHD患者的营养 状况, 提高MHD患者的PA值, 最终改善MHD患者的预后。

\section{2. 方法}

\section{1. 一般资料}

本研究采用单中心、随机、单剂量、单盲对照设计, 经成都市第五人民医院医学伦理委员会审批同意（No. 【2018】第118号），选取2018年3月一2019年8月在本院 接受血液透析治疗的终末期肾病患者 64 例。其中男性 34 例, 女性 30 例, 年龄 48 72岁, 平均57.0 4 .1岁。试验组 32 例, 年龄33 71岁, 平均年龄 $(50.3 \pm 10.4$ 岁); 对照组 32 例, 年龄35 72岁, 平均年龄 (51.8 \pm 9.9 ) 岁, 两组间 年龄、性别差异无统计学意义 $(\mathrm{P}>0.05)$ 。

\section{2. 纳入标准}

(1)符合1992年黄山会议座谈纪要中拟定的尿毒症诊 断标准, 或美国肾脏病基金会 $\mathrm{K} / \mathrm{DOQI}$ 专家组对慢性肾脏 病 (CKD) 分期建议中的CKD5 期的诊断标准; (2)维持血 液透析时间超过 3 个月; (3)除肾脏外无其它明显器质性病 变; (4)自愿签署知情同意书。

\section{3. 排除标准}

近3月来有严重心脑血管并发症、严重肺部、肝病疾 病等。(2)已行甲状旁腺切除者; (3)不能配合检查者。(4)对 本品及亮氨酸过敏者及高过敏体质人群。

\section{4. 研究方法}

试验组在透析期间三餐后予以HMB $2 \mathrm{~g} /$ 次 [10], 3次/ 天, 共 12 周; 对照组在透析期间三餐后后予以安慰剂 $2 \mathrm{~g} /$ 次, 3次/天, 共12周。

\section{5. 观察指标}

\subsection{1. 人体成分分析仪使用}

用生物电阻抗法通过体成分分析仪Inbody S770 (韩 国Biospace）来测定体成分。受检者进食2小时以上, 透 析后30分钟内测量, 无剧烈活动、输液等。排空大小便, 取下身上金属物品, 仅穿内衣、裤, 脱掉鞋袜, 消毒足 底后按照足形电极的形状踩在电极上, 脚后跟对牢原点, 自然直立站立。双手各握住手柄, 拇指按在手柄上方的 电极上, 四指握在手柄下方的电极上, 手臂自然伸直, 肘部无夹角, 与腋前线呈 $15^{\circ}$ 角。保持上诉站立姿势直 到测试结束。测试过程中, 手脚不得离开电极, 身体不 要随意晃动, 暂时保持不说话。观察两组患者相位角的 变化。

\subsection{2. 营养评分表}

观察两组患者干预前后各项指标, 所有患者均在入组 及出组 $48 \mathrm{~h}$ 内完成SGA评分、MIS评分, 参与调查的医护 人员调查前均进行相关培训。

\section{6. 不良反应观察}

若患者使用期间出现恶心、呕吐, 腹痛等胃肠道反应 时, 密切观察患者情况, 根据情况给予相应处理, 必要时 予以停用。

\section{7. 统计学处理}

\subsection{1. 差异性分析}

本次调查中, 采用配对样本 $\mathrm{t}$ 检验和独立样本 $\mathrm{t}$ 检验, SGA、MIS、PA实验组和对照组、前侧和后侧上的是否存 在差异是否存在差异。其中, 配对样本t检验适用于同一 组受试者接受前后两次测验时的两次测验值平均的差异 比较; 独立样本 $\mathrm{t}$ 检验统计法适用于两个平均数的差异检 验, 其适用的为自变量为二分间断变量、因变量为连续性 变量。

\subsection{2. 相关分析}

Pearson 相关系数是用来衡量两个定距变量间的线性 关系。相关系数的值在 -1 到 1 之间, 其绝对值越大, 表明 两者的相关性越强。相关系数越接近于 1 或-1, 相关度越 强, 反之则越弱。此外, 判断相关关系需要综合考虑相关 系数和显著性水平, 只有在相关系数大于 0 且显著性水平 Sig. $<0.05$ 的情况下才能说明变量之间是相关的。因此本文 采用Pearson相关系数检验实验组治疗后SGA、MIS、PA 之间是否存在相关。 
3. 结果

\section{1. 受试者流程（图1）}

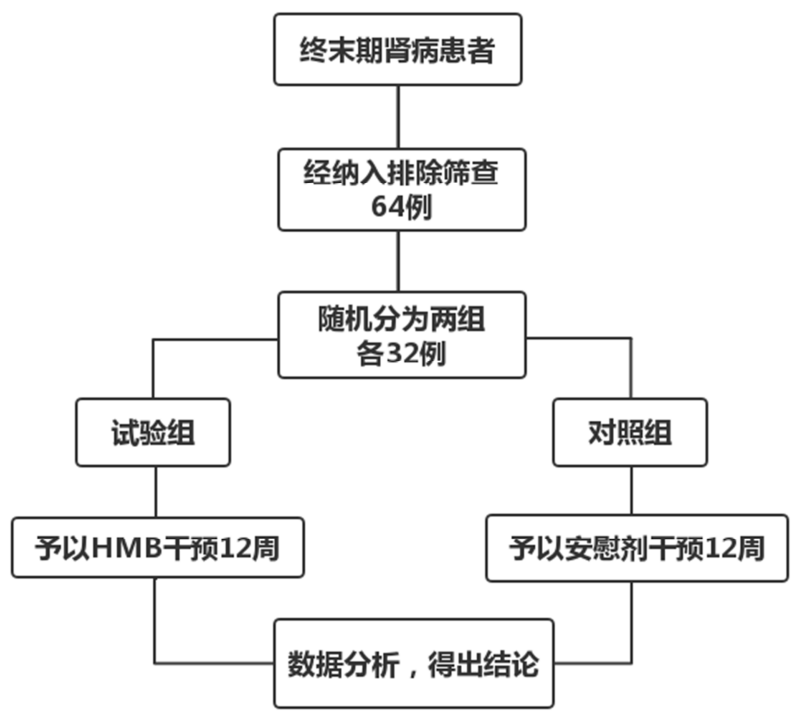

图1 受试者流程图。

\section{2. 两组患者干预前后相位角比较（表1）}

与本组干预前比较, 试验组干预后相位角升高明显, 有统计学意义 $(\mathrm{P}<0.05)$; 试验组升高幅度优于对照组, 有统计学意义 $(P<0.05)$ 。

表1 两组患者干预前后相位角(PA)比较。

\begin{tabular}{llll}
\hline 组别 & $\mathbf{N}$ & & PA \\
\hline \multirow{2}{*}{ 实验组 } & \multirow{2}{*}{32} & 治疗前 & $5.644 \pm 1.048$ \\
& & 治疗后 & $6.406 \pm 0.909 * \Delta$ \\
对照组 & \multirow{2}{*}{32} & 治疗前 & $5.697 \pm 0.996$ \\
& & 治疗后 & $5.659 \pm 0.919$ \\
\hline
\end{tabular}

注: 与本组治疗前比较, ${ }^{*} \mathrm{p}<0.05$; 与对照组同期比较, ${ }^{\Delta} \mathrm{p}<0.05$

\section{3．两组患者干预前后SGA营养评分比较（表2）}

与本组干预前比较, 试验组干预后 SGA评分降低, 有 统计学意义 $(\mathrm{P}<0.05)$; 试验组优于对照组, 有统计学意 义 $(P<0.05)$ 。

表2 两组患者干预前后主观全面评定(SGA)比较。

\begin{tabular}{|c|c|c|c|}
\hline 组别 & $\mathbf{N}$ & & SGA \\
\hline \multirow{2}{*}{ 实验组 } & \multirow{2}{*}{32} & 治疗前 & $5.281 \pm 4.191$ \\
\hline & & 治疗后 & $3.375 \pm 3.462 * \Delta$ \\
\hline \multirow{2}{*}{ 对照组 } & \multirow{2}{*}{32} & 治疗前 & $6.188 \pm 4.741$ \\
\hline & & 治疗后 & $5.594 \pm 4.110$ \\
\hline
\end{tabular}

注: 与本组治疗前比较, $* \mathrm{p}<0.05$; 与对照组同期比较, $\Delta \mathrm{p}<0.05$

\section{4．两组患者干预前后MIS营养评分比较（表3）}

与本组干预前比较, 试验组干预后MIS评分降低, 有 统计学意义 $(\mathrm{P}<0.05)$; 试验组优于对照组, 有统计学意 义 $(P<0.05)$ 。
表3 两组患者干预前后营养不良炎症评分(MIS)比较。

\begin{tabular}{llll}
\hline 组别 & $\mathbf{N}$ & MIS \\
\hline \multirow{2}{*}{ 实验组 } & \multirow{2}{*}{32} & 治疗前 & $13.406 \pm 8.609$ \\
& \multirow{2}{*}{ 对照组 } & 治疗后 & $7.281 \pm 5.782 * \Delta$ \\
& \multirow{2}{*}{32} & 治疗前 & $11.781 \pm 7.975$ \\
& & 治疗后 & $11.594 \pm 6.564$ \\
\hline
\end{tabular}

注: 与本组治疗前比较, $* \mathrm{p}<0.05$; 与对照组同期比较, ${ }^{\Delta} \mathrm{p}<0.05$

\section{5. 干预后相位角和SGA、MIS相关性（表4）分析}

从表 4 可以看出, $\mathrm{SGA}$ 与 MIS、PA的P值均小于 0.05 , 说明存在显著的正相关关系，相关的大小分别为 0.473 、 0.440 , 说明SGA表现水平越高, MIS、PA表现水平越高。 MIS与PA的P值均小于 0.05 , 说明存在显著的正相关关系, 相关的大小分别为 0.470 , 说明MIS表现水平越高, PA表 现水平越高。

表4 相位角(PA)和主观全面评定(SGA)、营养不良炎症评分(MIS)相关性 分析。

\begin{tabular}{lllll}
\hline & & SGA后 & MIS后 & PA后 \\
\hline \multirow{4}{*}{ SGA后 } & Pearson相关性 & 1 & $.473^{* *}$ & $.440^{*}$ \\
& Sig. (双尾) & & 0.006 & 0.012 \\
& N & 32 & 32 & 32 \\
\multirow{5}{*}{ MIS后 } & Pearson相关性 & $.473^{* *}$ & 1 & $.470^{* *}$ \\
& Sig. (双尾) & 0.006 & & 0.007 \\
& N & 32 & 32 & 32 \\
\multirow{2}{*}{ PA后 } & Pearson相关性 & $.440^{*}$ & $.470^{* *}$ & 1 \\
& Sig. (双尾) & 0.012 & 0.007 & \\
& $\mathrm{~N}$ & 32 & 32 & 32 \\
\hline
\end{tabular}

注: ${ }^{*} \mathrm{p}<0.05, * * \mathrm{p}<0.01$

\section{6. 不良反应}

HMB为白色偏苦味粉剂, 患者在治疗过程中, 初始 试验组有 1 例出现口感不适, 调整患者餐后服用后缓解; 余患者均未发现恶心、呕吐、腹胀、腹泻等不良反应。

\section{4. 讨论}

2008年, 国际肾脏营养与代谢学会提出“蛋白质-能量 消耗(PEW)”, 定义各种原因导致慢性肾病(CKD)患者蛋白 质能量储备降低的“营养不良”状态, 并制订了 PEW的诊断 标准, 提高了临床医师对CKD患者营养不良的认识[11]。 当人体因各种原因导致营养素摄入不足、不能满足机体需 要或利用障碍时, 就会导致营养不良和机体抵抗力下降。 评估和管理营养不良特别重要, 因为它可以改善病人的预 后。评价患者营养状况, 通常会通过体格检查、实验室检 测、营养状况评估量表等方式[12]。但每种方法都有局限 性 [12], 并且MHD患者的PEW 是多因素的, 并且是复杂的。 如SGA广泛用于评估透析患者的营养状况[13], 作为综合 的评分系统, 优于生化单一指标对营养状况的评估[14], 但存在明显的主观性, 易受并发症的干扰。MIS 是评估 MHD患者营养状况和识别PEW的有用工具 [15], 与人体测 量学指标、炎性指标和营养相关生化指标的相关性均高于 SGA, 是患者住院率和病死率的有效预测因子 [16]; 此外 
还可用于评估和预测CKD的并发症[17], 但其仍有部分主 观性。

相位角 (PA) 可以反映细胞膜的能力、细胞内外水 分的分布情况和体细胞量 [18], 其精确性不依赖人体测量 和公式 $[19]$ 。由于 $P A$ 是采用电阻 $(\mathrm{R})$ 和容抗 $(\mathrm{Xc})$ 的数据, 通 过固定公式计算得出, 所以其受机体液体分布影响小。年 龄、性别、BMI是健康成人PA值的决定因素, 在即状态下, $\mathrm{PA}$ 值低于健康人, 目前还没有研究证实哪些因素可以改 变患者的PA值。

补充HMB可以改善蛋白质平衡[20], HMB与其他营养 物质的联合应用已被用于临床治疗肌肉消耗, 包括 $\operatorname{COPD}[21]$ 、肿瘤[22]、艾滋病[23]等慢性疾病造成的肌肉 消耗。细胞实验显示HMB可以减少蛋白质的降解, 增加 蛋白质的合成[6], 其机制可能与通过减少炎症反应来增加 蛋白的合成, 激活雷帕霉素靶蛋白 (mTOR) 增加蛋白质 转化, 增加胰岛素生长因子 (IGF-1) 等有关。此外, HMB 也可能通过减弱半胱天冬酶的活性及负调节泛素-蛋白酶 体通路来减少蛋白质分解。另外, HMB还可能通过增加 卫星细胞活性, 从而增强肌肉再生能力 [6]。

本研究可以看出, 患者MIS、SGA的相关性成正相 关。PA能反映MHD患者的营养状况 [24], 可以早期识别 除出血白蛋白在正常范围而有潜在营养不良的患者 [25], 相位角是许多疾病的临床进展的预测指标, 与临床结局 密切相关[26], 低PA患者其住院时间延长 [27], 生存期缩 短, 死亡风险升高[28]; 并且PA是无创、快速、简单、 独立的定量评估患者营养状况的方法[29]。本研究可以看 出, 患者PA与MIS、SGA的相关性成正相关。PA值越大, 完整细胞膜越多, 细胞功能越强 [30], 此改变与Farias CL 等人 [31]发现一致。因为PA值的大小与营养不良、疾病 进展、病情预后等密切相关, 通过干预手段提高患者PA 值, 从而改善患者病情, 提高患者生存率和质量, 是非 常值得我们进一步研究的方向, 也是此次我们该研究的 最大目的。从表1结果中, 我们的研究也已证明, 予以补 充HMB可以提高患儿的PA值。通过对维持性血液透析患 者给予补充 $\mathrm{HMB}$, 可以改善患者营养状况, 提高患者相 位角, 改善患者预后; 且相位角提升程度和营养状况成 正相关。

\section{5. 结论}

维持性血液透析患者的预后往往与人体的营养状况有 关, 相位角 (PA) 能够在一定程度上反应细胞的健康和营 养状况。PA在临床上, 已被用于其他疾病的研究, 但很少 用于血透患者。经过我们的研究可以得出, PA可以成为透 析患者临床结果的预测指标之一, 可早期发现维持性血透 患者的营养不良。而我们创新性的给予维持性血液透析患 者补充HMB, 通过营养干预提高患者PA值, 从而早期干预、 早期改善病人的营养状况, 最终改善临床结局。

但此项研究有一定局限性, 因为这是一项单中心研究, 包括相对较少的患者。因此, 未来需要更大的前瞻性研究, 以确定PA在ESRD患者中的临床意义。

\section{致谢}

本文为四川省卫生计生委重点研究项目《血液净化治 疗联合 $\beta$-差弪基- $\beta$ 甲基丁酸盐（HMB）对尿毒症患者钻磷代 谢紊乱的疗效观察》(18ZD050)的阶段性成果之一。

\section{参考文献}

[1] Kim EJ, Choi MJ, Lee JH, et al.Extracellular FluidIntracellular Fluid Volume Ratio as a Novel Risk Indicator for All-Cause Mortality and Cardiovascular Disease in Hemodialysis Patients[J].PLOS ONE, 2017, 7(18):17-27.

[2] Rimsevicius L, Gincaite A, Vicka V, et al.Malnutrition assessent in hemodialysis patients: role of bioelectrical impedance analysis phase angle[J].J Ren Nutr, 2016, 26(6):391-395.

[3] Arnaud-Battandier F, Malvy D, Jeandel C, et al.Use of oral supplements in malnourished elderly patients living in the community: a pharmaco-economic study[J].Clin Nutr, 2004, 23(5):1096-1103.

[4] Waal D, Heaslip E, Callas P.Medical Nutrition Therapy for Chronic Kidney Disease Improves Biomarkers and Slows Time to Dialysis[J].J Ren Nutr, 2016, 26(1):1-9.

[5] Wilson JM, Lowery RP, Joy JM, et al.The effects of 12 weeks of beta-hydroxy-beta-methylbutyrate free acid supplementation on muscle mass,strength,and power in resistance-trained individuals: a randomized,double-blind,placebo-controlled $\operatorname{study}[\mathrm{J}]$.Eur $\quad \mathrm{J}$ Appl Physiol, 2014, 114(6):1217-1227.

[6] Fitschen PJ, WilsonGJ, Wilson JM, et al.Efficacy of $\beta$-hydroxy- $\beta$-methylbutyrate supplementation on elderly and clinical populations[J].Nurition, 2013, 29(1):29-36.

[7] Stegel P, Kozjek NR, Brumen BA, et al.Bioelectrical impedance phase angle as indicator and predictor of cachexia in head and neck cancer patients treated with(chemo) radiotherapy[J].Eur J Clin Nutr, 2016, 70(5):602-626.

[8] Zouridakis A, Simos YV, Verginadis H, et al.Correlation of bioelectrical impedance analysis phase angle with changes in oxidative stress on end-stage renal disease patients, before, during, and after dialysis[J].Ren Fail, 2016, 38(5):738-743.

[9] 黎晓偣. 应用生物电阻抗法评估血液透析病人参与肾功能 [J].实用医学杂志, 2015, 31(5):770-774。

[10] Nissen S, Sharp R, Ray M, et al.Effect of leucine metabolite beta-hydroxy-beta-methylbutyrate on muscle metabolism during resistance-exercise training[J].J Appl Physiol, 1996, 81(5):2095-2104.

[11] Fouque D, Kalantar ZK, Kopple J, et al.A proposed nomenclature and diagnostic criteria for protein-energy wasting in acute and chronic kidney disease[J].Kidney Int, 2008, 73(4):391-398. 
[12] Popovic V, Zerahn B, Heaf JG.Comparison of dual energy $\mathrm{x}$-ray absorptiometry and bioimpedance in assessing body composition and nutrition in peritoneal dialysis patients[J].J Ren Nutr, 2017, 27(5):355-363.

[13] Kopple JD.Clinical Practice Guidelines for Nutrition in Chronic Renal FailureKDOQI,National Kidney Foundation[J].Am J Kidney Dis, 2000, 35(6 Suppl 2):1-140.

[14] Espahbodi F, Khoddad T, Esmaeili L.Evaluation of Malnutrition and Its Association With Biochemical Parameters in Patients With End Stage Renal Disease Undergoing Hemodialysis Using Subjective Global Assessment[J].Nephrourol Mon, 2014, 6(3):250-321.

[15] Gonzalez OAJ, Arce SCV, Vega VO, et al.Assessment of the Reliability and Consistency of the "Malnutrition Inflammation Score" (MIS) in Mexican Adults With Chronic Kidney Disease for Diagnosis of Protein-Energy Wasting Syndrome (PEW)[J].Nutr Hosp, 2014, 31(3):1352-1358.

[16] Afsar B, Sezer S, Ozdemir FN, et al.Malnutrition-inflammation Score Is a Useful Tool in Peritoneal Dialysis Patients[J].Perit Dial Int, 2006, 26(6):705-711.

[17] Ho LC, Wang HH, Chiang CK, et al.Malnutrition-inflammation Score Independently Determined Cardiovascular and Infection Risk in Peritoneal Dialysis Patients[J].Blood Purif, 2010, 30(1):16-24.

[18] Ruiz-Margain A, Macias-Rodriguez RU, Duarte-Rojo A, et al.Malutrition assessed through phase angle and its relation to prognosis in patients with compensated liver cirrhosis: a prospective cohort study[J].Dig Liver Dis, 2015, 47(4):309-314.

[19] 宁华英, 赵崇法, 齐玉梅, 等.相位角预测手术病人营养风 险及住院时间 [J].肠外肠内营养, 2017, 24(5):296-300。

[20] Jowko E, Ostaszewski P, Jank $M$, et al.Creatine and Beta-Hydroxy-Beta-Methylbutyrate (HMB) Additively Increase Lean Body Mass and Muscle Strength During a Weight-Training Program[J].Nutrition, 2001, 17(7-8):558-566.

[21] Hsieh LC, Chien SL, Huang MS, et al.Anti-inflammatory and anticatabolic effects of short-term, beta-hydroxy-beta-methylbutyratesupplementation on chronic obstructive pulmonary disease patients inintensive care unit[J].Asia Pac J Clin Nutr, 2006, 15(14):544-550.
[22] Berk L, James J, Schwartz A, et al.A Randomized,Double-Blind,Placebo-Controlled Trial of a Beta-Hydroxyl Beta-Methyl Butyrate,Glutamine, and Arginine Mixture for the Treatment of Cancer Cachexia (RTOG 0122)[J].Support Care Cancer, 2008, 16(10):1179-1188.

[23] Rathmacher JA, Nissen S, Panton L, et al.Supplementation with a combination of (HMB), arginie, and glutamine is safe and could improve hematological parameters[J].PEN J Parenter Enteral Nutr, 2004, 28(2):65-75.

[24] Lee JY, Kim JS, Yang JW, et al.Serum leptin level is associated with phase angle in CKD5 patients not undergoing dialysis . [J].PLoS ONE, 2018, 13(8):20-25.

[25] Wagner D, Adunka C, Kniepeiss D, et al.Serum albumin,subjective glbal assessment,body mass index and the bioimpedance analysis in the assessment of malnutrition in patients up to 15 years after liver transplantation[J].Clin Transplant, 2011, 25(4):396-400.

[26] Belarmino G, Gonzalez MC, Torrinhas RS, et al.Phase angle obtained by bioelectrical impedance analysis independently predicts mortality in patients with cirrhosis[J].World J Hepatol, 2017, 9(7):401-408.

[27] Varan HD, Bolayir B, Kara O, et al.Phase angle assessment by bioelectrical impedance analysis and its predictive value for malnutrition risk in hospitalized geriatric patients[J].Aging Clin Exp Res, 2016, 28(6):1121-1126.

[28] Peres WA, Lento DF, Baluz K, et al.Phase angle as a nutritional evaluation tool in all stages of chronic liver disease[J].Nutr Hosp, 2012, 27(6):2072-2078.

[29] Gabriela LE, Iván AOP. Phase angle and mid arm circumference as predictors of protein energy wasting in renal replacement therapy patients[J].Nutr Hosp, 2019, 36(3):633-639.

[30] Ceniccola GD, Castro MG, Piovacari SM, et al.Current technologies in body composition assessment: advantages and disadvantages[J].Nutririon, 2019, 62(1):25-31.

[31] Farias CL, Campos DJ, Bonfin CM, et al.Phase angle from $\mathrm{BIA}$ as a prognostic and nutritional status stool for children and adolescents undergoning hematopoietic stem cell transplantation[J].Clin Nutr, 2013, 32(3):420-425. 